Kalpa Publications in Engineering
volume 3, 2020, Pages 270-278
Proceedings of International Sym-
posium on Applied Science 2019

\title{
Evaluation Of Vascular Disorder Effect On Bioimpedance Values Using Finite Element Method
}

\author{
Anh Vy Ngo Hoang ${ }^{1}$, Thu Uyen Le Thi ${ }^{1}$, Quang Linh Huynh ${ }^{1}$, Son Thuy \\ Nguyen $\mathrm{Nhu}^{1}$ \\ Ho Chi Minh City University of Technology - VNUHCM \\ 1614194@hcmut.edu.vn
}

\begin{abstract}
Introduction: Cardiovascular diseases are the first cause of death globally: more people die annually from these diseases than from any other causes. These diseases are a group of disorders of the heart and blood vessels that appears silently but lead many serious consequences for life. Vascular diseases including: atherosclerosis, aneurysm, peripheral artery disease... affect the arteries, veins, or capillaries throughout the body and around the heart. Therefore, assessment the quality of blood vessels early is extremely necessary in order to prevent cardiovascular diseases. Bioimpedance analysis is a non-invasive, safe and low cost technique which is widely used for diagnosis various diseases. This study conducts to a novel method to evaluate the status of blood vessel in two cases: stenosis and aneurysm based on bioimpedance signal. A 3D model of forearm with several layers (skin, fat, muscle, bone and blood vessel) and electrodes is simulated by using Comsol Multiphysics software. The electric impedance in different vascular conditions is examined. The results show that vessel impedance changed substantially because of vascular disorders. The bioimpedance signals vary according to the sizes of plaque and aneurysm wall. Consequently, bioimpedance analysis is reliable to detect vascular diseases and able to be applied in near future.
\end{abstract}

\section{Introduction}

The cardiovascular system comprises the heart, and blood vessels, that is arteries, arterioles, capillaries, venules and veins, which enclose and distribute blood to the tissues. There is a number of clinical disorders associated with arteries and veins. These include peripheral arterial disease, arterial aneurysms and dissections, strokes, varicose veins and deep vein thromboses, which lead to the death quickly [1]. According to the report of World Health Organization, in the 2016 global ten main death causes, vascular diseases such as ischemia heart disease and stroke were the first two [2]. 
There are various reasons of cardiovascular disease. The main reasons are atherosclerosis and aneurysms. An atherosclerosis is formed when a substance called plaque builds up in the walls of the arteries. The plaque is a deposit combined of cholesterol, macrophages, calcium, or other substances from the blood. This deposit develops thicker and interferes with blood flow through the arteries. A heart attack or stroke can happen when a blood clot forms and block the blood flow [3]. As far as the weak areas of an arterial wall is concerned, it finds easy to develop an aneurysm, which is a round or tube-like bulge. Frequently, there are no symptoms, but at their most severe stage, some can rupture, leading to life-threatening internal bleeding, then the patient will typically go into severe shock [4]. Aortic rupture is usually fatal. The treatment of ruptured aneurysms involves surgical repair, which is extremely risky [1]. Thus, in turn, a early diagnosis is extremely necessary. Then the surrogate endpoints can be delayed and even reversed by lifestyle and drug intervention.

In this day and age, when health is the top concern of global people and the fact of such a huge disease burden, prevention and treatment of vascular related diseases are equally important. There are many state of the art techniques to diagnose these diseases such as: blood test or imaging diagnosis like ultrasound, computed tomography and MRI. These methods are complicated and time consuming with high cost. Recently, bioelectrical impedance analysis (BIA) is a new modality in body composition estimation and evaluation of clinical conditions. Numerous researchers have conducted studies on bioimpedance analysis due to the non invasiveness, the low cost and the portability of bioimpedance analysis systems.

Bioimpedance analysis is a technique, that is broadly applied in body composition measurements and healthcare assessment systems. There is a wide spectrum of utilization of bioimpedance in healthcare facilities such as disease prognosis and monitoring of body vital status [5].

Bioimpedance or biological impedance is defined as the ability of biological tissue to impede electric current [7]. Early experiments in the 19th century established that biological tissues had passive (and active) electrical properties that differed greatly from an electrolytic solution [6].

A living object is developed with cells. Each biological cell containing intracellular fluids (ICF), cell membranes with or without cell wall and is suspended in the extracellular fluids (ECF). The cell membrane is made up hydrophilic (polar) heads and hydrophobic (nonpolar) tails which form phospholipid bilayer. This phospholipid bilayer provides a capacitance to the applied alternating current signal and contributes to a capacitive reactance. The intracellular fluids are compounds of the cytoplasm and the nucleus. The cytoplasm and the nucleus are mostly made up of solution of proteins, different chemicals, salts, and waters, which are electrically conducting. The extracellular fluids are also made up of electrically conducting materials. As the intracellular fluids and extracellular fluids in biological tissues are composed of ionic solution and the highly conducting materials, they provide highly conducting paths (low resistive paths) to the applied current signal [7].

In bioimpedance analysis, the scientists inject an alternating current $(I)$ into the object and use the electrode to collect the voltage $(V)$. Mathematically, dividing the voltage signal measured $(V)$ by the current signal applied $(I)$ produces a complex bioelectrical impedance $(\mathrm{Z})$.

where:

$$
Z=R+X_{C} \cdot j
$$

$$
\begin{aligned}
& Z: \text { impedance }(\mathrm{Ohm}) \\
& R \text { : resistance }(\mathrm{Ohm}) \\
& X_{c}: \text { reactance }(\mathrm{Ohm})
\end{aligned}
$$

Resistance is the opposition to the flow of current when passing through the body. Reactance is the delay in conduction caused by cell membranes, tissue interfaces, and nonionic substances [8].

In tetrapolar electrodes configuration shown in Figure 1, two electrodes are attached to the body to inject the current flow $(I)$ and others called "sensing electrodes" are utilized to detect the voltage $(V)$ [9]. 
This study simulates blood vessels under different conditions to consider the impact of vascular disorders on bioimpedance. First, we investigated bioimpedance in normal vessel. Then, we added the plaque in the vessel and examined bioimpedance values when changing the size of plaque. Finally, in aneurysm case, we changed the size of aneurysm and measured bioimpedance across the sensing electrodes. All the measures are carried out in $50 \mathrm{kHz}$ frequency.

According to the values obtained, the study demonstrated the ability of using bioimpedance in order to detect vascular disorders. Thereby, it provides a novel method for diagnosing cardiovascular diseases.

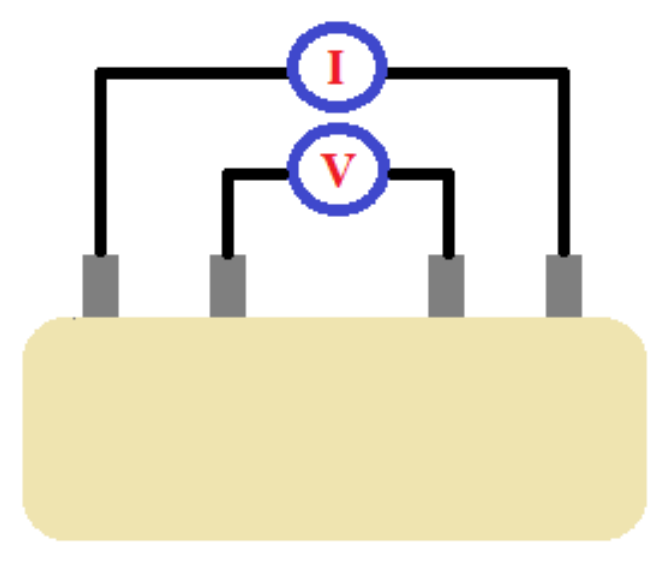

Figure 1. Configuration of tetrapolar electrodes

\section{Methods}

Finite element model of blood vessel model was developed using the AC/DC Module, Electric Current Physics, in Comsol Multiphysics software. For our study, we have considered the variation of the abnormal vessel size in the simplified forearm model.

Firstly, in terms of geometry, the forearm model is expressed as a composition of layers (skin, fat, muscles, bone, vessel, vessel wall). Each layer is described as a cylinder which length is $100 \mathrm{~mm}$. The dimension of each layer is defined by the following Table 1 . We used the tetrapolar electrodes configuration to measure the bioimpedance. The volume of electrodes are $5 \times 10 \times 1 \mathrm{~mm} 3$ and the centers of electrodes are placed alternately at $Z=12,5 \mathrm{~mm}, Z=42.5 \mathrm{~mm}, \mathrm{Z}=57.5 \mathrm{~mm}$ and $\mathrm{Z}=87.5 \mathrm{~mm}$ [9].

Table 1. The dimensions of organs in model

\begin{tabular}{|r|c|c|}
\hline \multicolumn{2}{|c|}{ Organ } & Radius \\
\hline 1 & Skin & $38 \mathrm{~mm}$ \\
\hline 2 & Fat & $35 \mathrm{~mm}$ \\
\hline 3 & Muscle & $25 \mathrm{~mm}$ \\
\hline 4 & Bone & $10 \mathrm{~mm}$ \\
\hline 5 & Blood & $1.5 \mathrm{~mm}$ \\
\hline 6 & Vessel wall & $1.7 \mathrm{~mm}$ \\
\hline
\end{tabular}




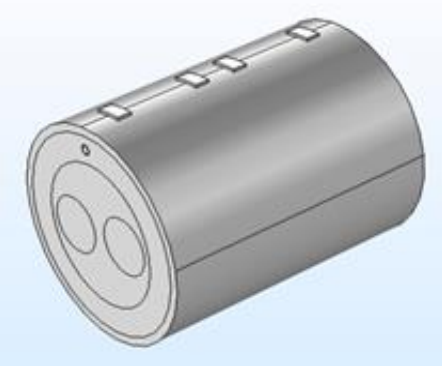

Figure 2. The forearm model in COMSOL Multiphysics

\subsection{Atherosclerosis case:}

The position of plaque center is placed at $\mathrm{Z}=50 \mathrm{~mm}$ in the lumen blood. To examine the impact of the plaque on bioimpedance, bioimpedance was measured at different conditions of plaque shape. Initially, length of the plaque was kept unchanged at $20 \mathrm{~mm}$ and height is set from $0.5 \mathrm{~mm}$ to $3 \mathrm{~mm}$ in increment of $0.5 \mathrm{~mm}$ in each measurement. Following, height of the plaque was kept unchanged at 1 $\mathrm{mm}$ and length was set from $2 \mathrm{~mm}$ to $20 \mathrm{~mm}$ in increment of $2 \mathrm{~mm}$ in each measurement [9].

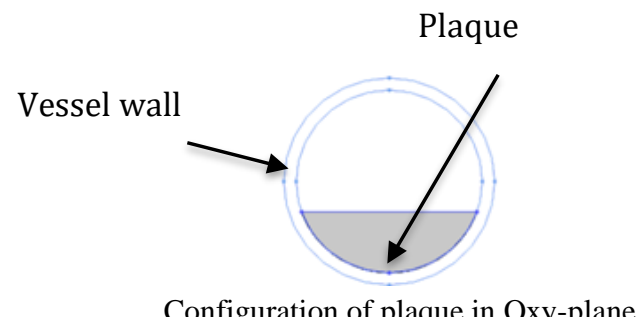

\subsection{Aneurysm case:}

Like the atherosclerosis case, the center of aneurysm is located at $\mathrm{Z}=50 \mathrm{~mm}$, which is located between the two voltage electrodes. Wall swelling size was changed to survey how volatile the impedance was. Initially, the saccular aneurysm height varies from $0.5 \mathrm{~mm}$ to $3 \mathrm{~mm}$ while the length is $20 \mathrm{~mm}$. Regarding the erratic length case, height was unchanged with $1 \mathrm{~mm}$ while wall swelling was presented in different lengths from $2 \mathrm{~mm}$ to $20 \mathrm{~mm}$ [9].

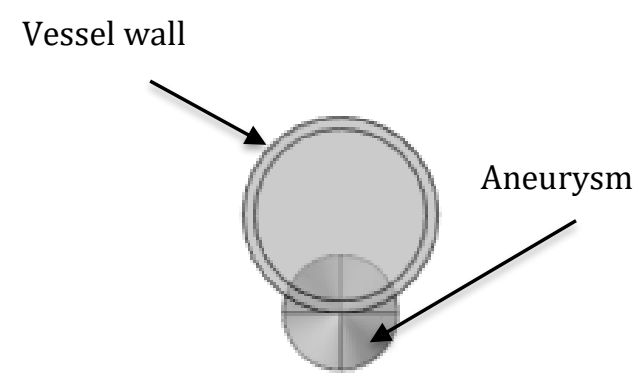

Figure 4. Configuration of plaque in Oxy-plane 
Secondly, the Materials: skin, fat, muscle, bone, blood, vessel wall, copper for electrodes and plaque. Each material is described as set of features at $50 \mathrm{kHz}$ as shown in Table 2 [10].

Table 2. The dielectric properties of tissues at $50 \mathrm{KHz}$ [9]

\begin{tabular}{|c|c|c|c|}
\hline \multicolumn{2}{|c|}{ Organ } & Permittivity & $\begin{array}{c}\text { Conductivity } \\
(\mathrm{S} / \mathrm{m})\end{array}$ \\
\hline 1 & Skin & 0.029 & 0.029 \\
\hline 2 & Fat & 0.025 & 0.025 \\
\hline 3 & Muscle & 10094 & 0.35 \\
\hline 4 & Bone & 613 & 0.083 \\
\hline 5 & Blood & 5197 & 0.7 \\
\hline 6 & Vessel & 1633 & 0.316 \\
\hline 7 & Wopll & & 1 \\
\hline 8 & Plaque & 172 & 0.02 \\
\hline
\end{tabular}

Thirdly, electrical current module was applied in Physics session. The outer two electrodes were used for applying $1 \mathrm{~mA}$ electrical signal (boundary current source) and ground.

Next, in Meshing session, "finer" element size was chosen to enhance the accuracy of the model.

Finally, the numerical simulations was performed in $50 \mathrm{kHz}$ and we extracted the bioimpedance across the sensing electrodes to investigate.

\section{RESULTS}

In this part, we present the results in two categories: Atherosclerosis case and Aneurysm case. In every case, we compute the relation of measured bioimpedances between abnormal and normal vessel (ZS / ZN, ZA / ZN), where ZN, ZS and ZA are the impedance values in the normal, plaque and aneurysm cases.

\subsection{Atherosclerosis case}

As previously mentioned, in atherosclerosis, we performed 2 different conditions: changing height and changing length of plaque.

First, the bioimpedance values were extracted when changing the height plaque from $0.5 \mathrm{~mm}$ to 3 $\mathrm{mm}$. The results shown that when the height of plaque increases, the bioimpedance also rises. Concretely, the impedance values were increased by $0.75 \%, 2.11 \%, 3.85 \%, 5.84 \%, 7.77 \%$ and $8.96 \%$ for $0.5 \mathrm{~mm}, 1 \mathrm{~mm}, 1.5 \mathrm{~mm}, 2 \mathrm{~mm}, 2.5 \mathrm{~mm}$ and $3 \mathrm{~mm}$ respectively, as shown in Figure 4 .

Secondly, we found that the longer the plaque is, the higher the bioimpedance value is. The impedance values were increased by $0.44 \%, 0.73 \%, 0.99 \%, 1.23 \%, 1.44 \%, 1.63 \%, 1.79 \%, 1.92 \%$, $2.03 \%$ and $2.1 \%$ for $2 \mathrm{~mm}, 4 \mathrm{~mm}, 6 \mathrm{~mm}, 8 \mathrm{~mm}, 10 \mathrm{~mm}, 12 \mathrm{~mm}, 14 \mathrm{~mm}, 16 \mathrm{~mm}, 18 \mathrm{~mm}$ and $20 \mathrm{~mm}$ respectively, as shown in Figure 5.

\subsection{Aneurysm case}

Also, we present measures impedance in two cases of different aneurysm sizes:

Firstly, aneurysm height varies from $0.5 \mathrm{~mm}$ to $3 \mathrm{~mm}$ while length is $20 \mathrm{~mm}$. Secondly, the length wall swelling is varies from $2 \mathrm{~mm}$ to $20 \mathrm{~mm}$ while height is $1 \mathrm{~mm}$. 
As we can see in two figure 6 and 7, there is a negative correlation between the measured impedance and aneurysm sizes. In terms of the variation of aneurysm height, the results indicates that the impedance values were decreased by $0.5 \%, 1.9 \%, 4 \%, 6.5 \%, 9.4 \%, 12.5 \%$ for $0.5 \mathrm{~mm}, 1 \mathrm{~mm}, 1.5 \mathrm{~mm}$, $2 \mathrm{~mm}, 2.5 \mathrm{~mm}, 3 \mathrm{~mm}$ respectively, as illustrated in figure 6 . With regard to the variation of length, as shown in figure 7, the impedance were slipped steadily from $99.8 \%$ for $2 \mathrm{~mm}$ to $98.1 \%$ for $20 \mathrm{~mm}$.

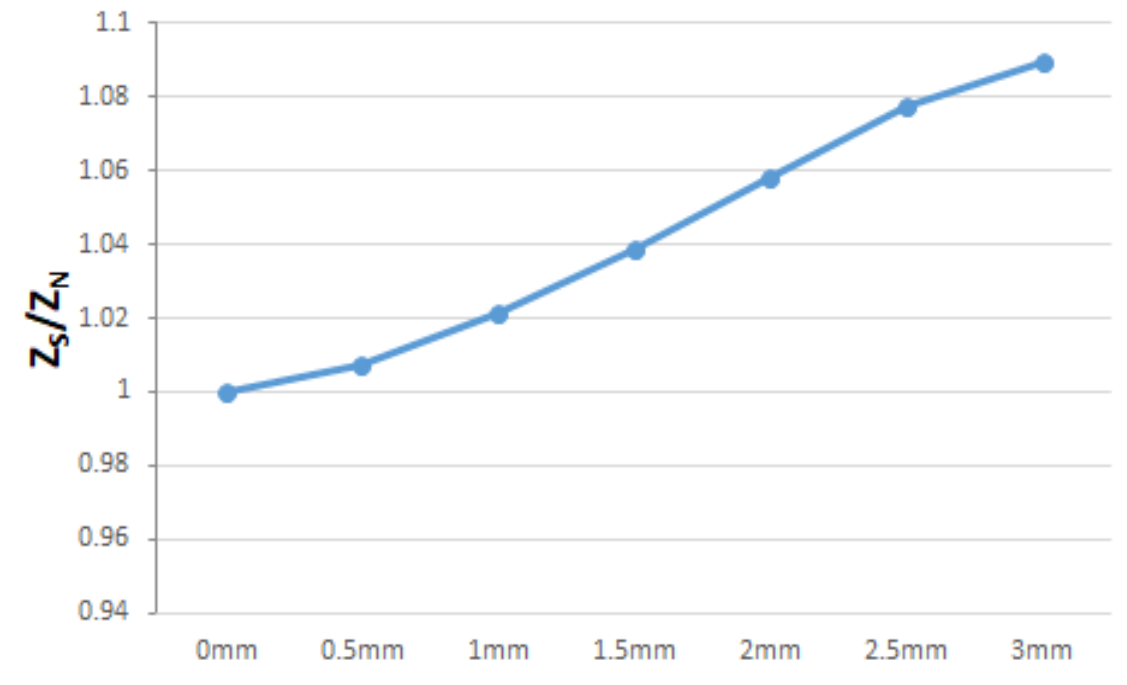

Plaque Height

Figure 5. The measured impedances at various height of plaque

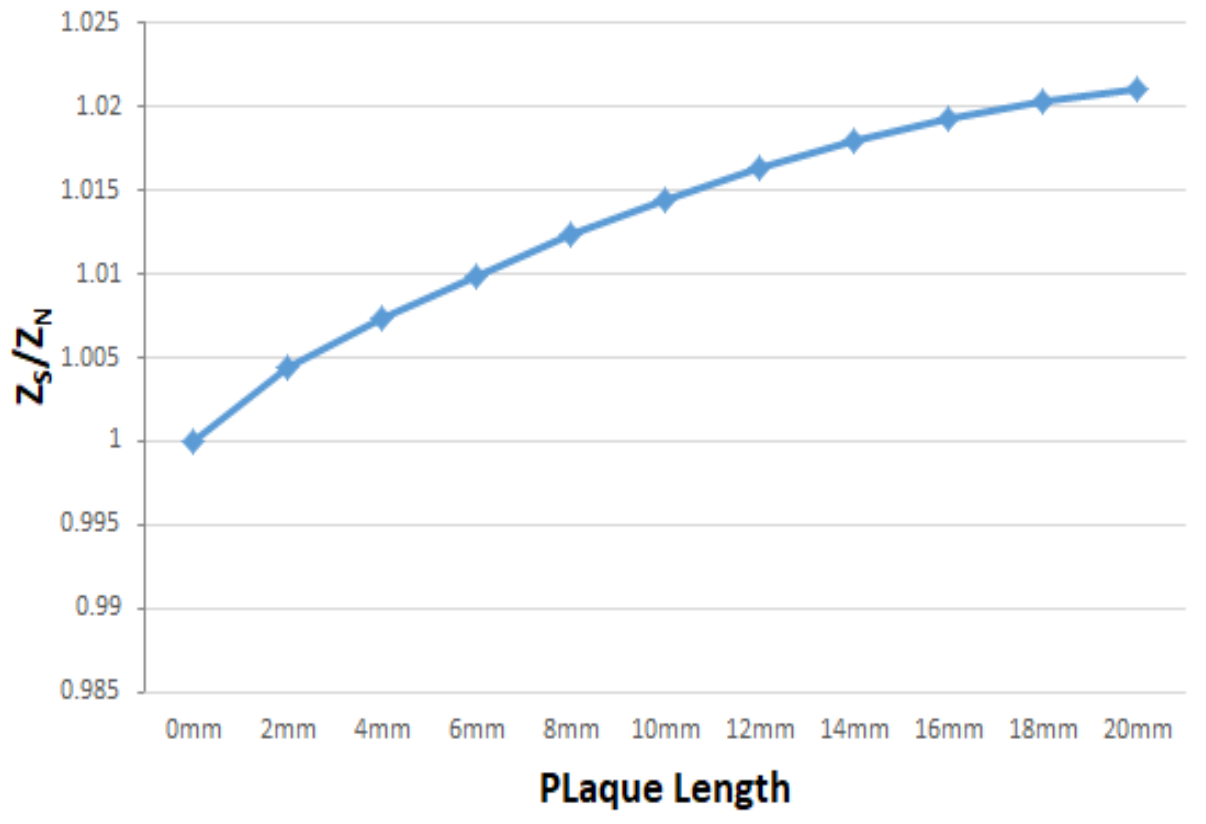

Figure 6. The measured impedances at various length of plaque 


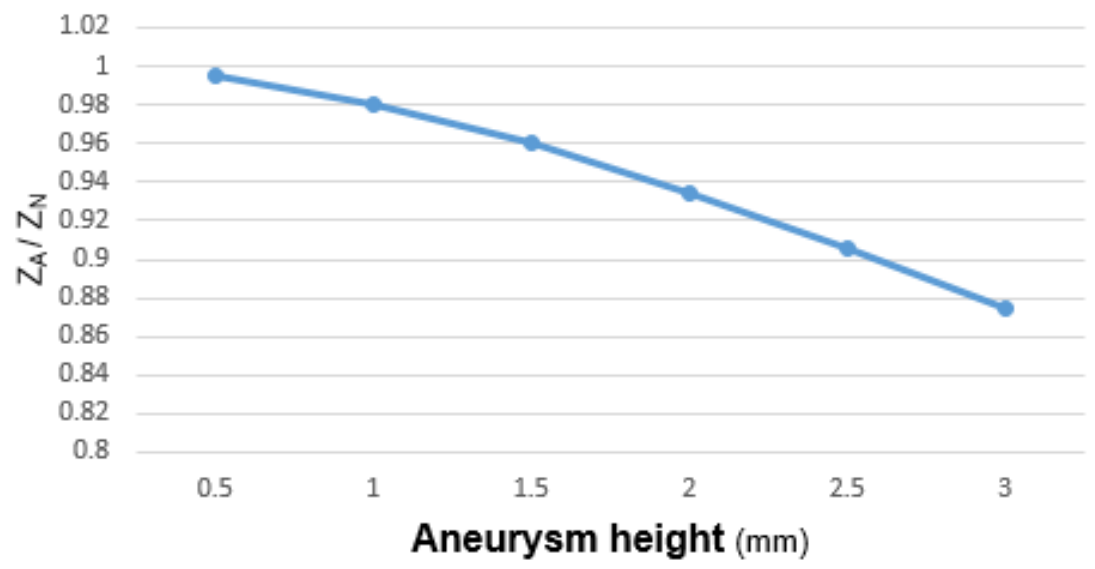

Figure 7. The measured impedances at various height of aneurysm

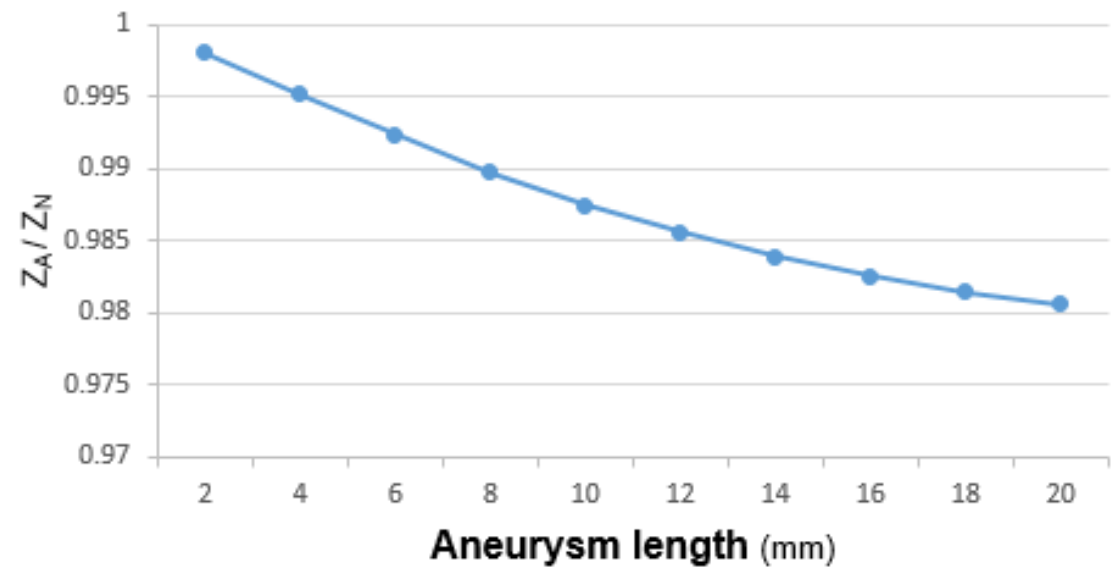

Figure 8. The measured impedances at various length of aneurysm

\section{Discussion}

Due to the danger of cardiovascular diseases, early evaluation of abnormal status and intervention to prevent or even reverse the occurrence of adverse vascular events have become a hot research topic in the world. In this research, we discuss a non invasive and simple method that can detect abnormal vessel. In fact, there are many research and clinical application of early assessment technology of vascular diseases: Doppler ultrasound is widely non-invasive, inexpensive method to diagnosis cardiovascular diseases. This technique uses Doppler effect to estimate the blood flow through the blood vessels by detecting the frequency shift of emitted frequency and the received frequency. However, the accuracy depends on operators, who need to be trained in intensive skills [11],[12]. Another technique assessing plaque composition accurately is computed tomography angiography (CTA). It allows simultaneous angiographic as well as anatomical volume acquisition in seconds, relatively low cost. The disadvantage of this technique is that the identification of liquid core and plaque bleeding is still lack of specificity. High resolution magnetic resonance imaging (MRI) is also sensitivity and specificity method to identify and measure plaque composition (lipid rich core, fibrous cap thickness, plaque 
bleeding and calcification, etc.), and detect vulnerable plaque. Long scan times and relative high cost, contraindications makes it difficult for everyone to apply [12],[13]. These aforementioned modalities have its own advantages however all of them are high cost, complicated and need extensive skill, while impedance measurement is not only easy to operate, but also low cost. This is a great method for the first step of diagnosing and assessing vascular diseases.

Cardiovascular diseases have previously been characterized as affecting "rich" countries, but in fact, a statistic proved that the number of these cardiovascular deaths occurred in low-income and middleincome countries is approximately similar in developed regions, even that there is a bounce in these countries [14]. Therefore, development of a low cost modality like BIA is very practical.

Electrical impedance of the forearm is estimated by measuring the voltage signal developed across that body part by injecting a constant current signal to the object. Mathematically, the impedance $(Z)$ is measured by dividing the voltage signal measured $(V)$ by the current signal applied $(I)$. As we explained above, $Z$ is complex quantity and it will have a particular phase angle $(\theta)$ depending on the tissue properties [7]. Hence, as we predicted, the impedance was increase in the stenosis case. This is simply because the main component of plaque is cholesterol which electrical conductivity was lower than blood. Therefore, the model was less conductive than normal case. In terms of aneurysm case, the swelling wall has the same electrical properties of blood then the impedance would have a decrease trend. As the aforementioned section, our simulation results also expressed that trend.

In atherosclerosis case, we found that the correlation coefficient between bioimpedance values and plaque sizes is significant (height case: $\mathrm{r}=0.994, \% \mathrm{RSD}=3.33 \%$ and length case: $\mathrm{r}=0.978, \% \mathrm{RSD}=$ $0.684 \%$ ). There is sufficient evidence to conclude there is a significant linear relationship between plaque shape and bioimpedance. Regarding to aneurysm case, the results illustrate that there is a strong negative correlation in the values of bioimpedance and heights of wall swelling. It is indicated by the correlation coefficient $(r=-0.992$ and -0.984$)$ and Relative Standard Deviation (RSD $=4.85 \%$ and $0.61 \%$ ) in the height cases and the length cases respectively. Hence we can conclude that the impedance signal varies based on the change of abnormal size. These proves that bioimpedance analysis can be utilized to diagnose vascular abnormalities. In general, in both of diseases cases, the variations of impedance are affected by the changes of height abnormal part more than the changes of length.

Although this study just simulated the blood vessel in the forearm, it provided positive results as a basis for future research. The prospect of this study is simulate the blood vessel in heart to examine directly heart diseases.

\section{Conclusion}

This research utilized bioelectrical impedance analysis technique to screen the vascular diseases. A model of non-invasive electrodes for measurement of blood vessel bioimpedance was constructed, used to simulate the variation of impedance under different vascular disease cases. As the abnormal session size getting larger, there is a moderate deviation of impedance from the normal case which is a good indication of BIA ability to identify the diseased vessel.

\section{References}

Ahmed N., Dawson M., Smith C. and Wood E. - Biology of Disease, Taylor \& Francis Group, Wilts, 2006, p. 407.

World Health Organization. 2018. The top 10 causes of death. [https://www.who.int/newsroom/fact-sheets/detail/the-top-10-causes-of-death]. Accessed August 5, 2018. 
American Heart Association. 2017. Atherosclerosis. [https://www.heart.org/en/healthtopics/cholesterol/about-cholesterol/atherosclerosis]. Accessed August 19, 2019.

MacGill M., 2017. Causes and treatments of aneurysm
[https://www.medicalnewstoday.com/articles/156993.php]. Accessed August 19, 2019.

Baumgartner R. N., Chumlea W. C. and Roche A. F. - Bioelectric impedance phase angle and body composition, Am. J. Clin. Nutr., 48 (1988) 16-23.

Cuba Gyllensten I.G.L. - Monitoring heart failure using noninvasive measurements of thoracic impedance, Eindhoven University of Technology, 2018, p.11.

Bera T. K. - Bioelectrical Impedance Methods for Noninvasive Health Monitoring: A Review, J. Med. Eng., 2014 (2014) 1-28.

Mulasi U., Kuchnia A. J., Cole A. J, and Earthman C. P. - Bioimpedance at the Bedside: Current Applications, Limitations, and Opportunities, Nutrition in Clinical Practice, 20 (2015) 1-14.

Shash Y. H., Eldosoky M. A. A. and Elwakad M. T. - The effect of vascular diseases on bioimpedance measurements: mathematical modeling, Biomed. Res. Ther., 5 (2018) 2414-2431.

Abdessalem K. B. and Salah R. B. - Diagnosis of arterial thrombosis and stenosis in blood vessel using bioimpedance analysis, International Research Journal of Engineering and Technology, 2 (2015) 316-321.

Al-Qaisi M., Nott D. M., King D. H., Kaddoura S. and Hamady M. - Imaging of peripheral vascular disease, Reports in Medical Imaging, 2 (2009) 25-34.

Barnes R. W. - Noninvasive diagnostic techniques in peripheral vascular disease, American Heart Journal, 97 (1979) 241-258.

Liu H. and Wang H. - Early Detection System of Vascular Disease and Its Application Prospect, BioMed Research International, 2016 (2016) 1-11.

Celermajer D. S., Chow C. K., Marijon E., Anstey N. M. and Woo K. S. - Cardiovascular Disease in the Developing World: Prevalences, Patterns, and the Potential of Early Disease Detection, Journal of the American College of Cardiology, 60 (2012) 1207-1216. 